\title{
Artigo/Article
}

\section{Prevalência da esquistossomose num povoado do Município de Tutóia, Estado do Maranhão}

\author{
Schistosomiasis prevalence in Tutóia village, Maranhão, Brazil
}

Ana de Matos Santos ${ }^{1}$ e Ana Carolina Fonseca Lindoso Melo ${ }^{1}$

\begin{abstract}
RESUMO
Introdução: A esquistossomose mansônica é uma patologia endêmica dos países subdesenvolvidos ou em desenvolvimento. O objetivo deste trabalho foi definir a prevalência da esquistossomose no povoado de Bom Gosto em Tutóia, Maranhão em 2008 e detectar a ocorrência do hospedeiro intermediário. Métodos: Foi realizado um levantamento dos dados das amostras de fezes analisadas pela Secretaria de Saúde Municipal e Fundação Nacional de Saúde. Em seguida, foram coletados e analisados 60 caramujos. A análise dos moluscos foi feita pelo processo de esmagamento. Quanto aos sedimentos fecais, usou-se o método de Kato-Katz. Resultados: De acordo com dados da Secretaria de Saúde Municipal e FUNASA, a prevalência da esquistossomose mansônica no povoado é de 3,2\%. Os 60 caramujos são da espécie Biomphalaria glabrata, e cinco (8,3\%) encontravam-se parasitados por Schistosoma mansoni. Conclusões: O povoado Bom Gosto é uma região de baixa prevalência da esquistossomose mansônica. A área investigada tem todos os componentes da cadeia epidemiológica de S. mansoni, explicando a ocorrência da doença na região.
\end{abstract}

Palavras-chaves: Estado do Maranhão. Esquistossomose. Schistosoma mansoni. Biomphalaria glabrata.

\begin{abstract}
Introduction: Schistosomiasis mansoni is an endemic disease in underdeveloped or developing countries. The study aimed to define schistosomiasis prevalence in Bom Gosto village, Tutóia, Maranhão, in 2008 and detect intermediate host occurrence. Methods: A survey of data from stool samples examined by the Municipal Department of Health and National Health Foundation was conducted. Next, 60 snails were collected and analyzed. Mollusk analysis was achieved by the crushing process. The Kato-Katz method was used to evaluate fecal sediment. Results: According to data from the Municipal Department of Health and FUNASA, schistosomiasis prevalence in the village was $3.2 \%$. The 60 snails were identified as Biomphalaria glabrata and five (8.3\%) were parasitized by Schistosoma mansoni. Conclusions: The Bom Gosto village is a low prevalence region of schistosomiasis mansoni. The study area has all the components of the epidemiological chain of S. mansoni, which explains the occurrence of disease in the region.
\end{abstract}

Keywords: State of Maranhão. Schistosomiasis. Schistosoma mansoni. Biomphalaria glabrata.

\footnotetext{
1. Departamento de Biomedicina, Setor de Parasitologia, Universidade Federal do Piauí, Parnaíba, PI. Endereço para correspondência: Dra. Ana Carolina Fonseca Lindoso Melo. Depto de Biomedicina/UFPI/ Campus Universitário Ministro Reis Veloso. Av. São Sebastião 2819, 64202-020 Parnaíba, PI. e-mail: carolinamelo@ufpi.br

Tel: 5586 9976-9820

Recebido para publicação em 29/03/2010

Aceito em 04/08/2010
}

\section{INTRODUÇÃO}

Schistosoma mansoni acomete de 2,5 a 6 milhões de pessoas no Brasil ${ }^{1}$, causando a esquistossomose. Esta doença ocorre principalmente em comunidades rurais, com expansão para os centros urbanos, estando intimamente relacionada com precárias condições higiênicas e inadequados recursos sanitários ${ }^{2}$.

Vários estados do nordeste brasileiro são endêmicos para esta parasitose, já tendo sido relatada em Pernambuco, Bahia, Maranhão ${ }^{2-4}$, bem como em Minas Gerais ${ }^{5}$ e São Paulo ${ }^{6}$ Logo, no Brasil, a esquistossomose ainda é um problema de saúde pública amplamente disseminado.

A ausência de dados precisos da prevalência da esquistossomose indica a necessidade de adequado levantamento nacional desta importante endemia parasitária ${ }^{1}$. No Maranhão, as estimativas da frequência desta doença indicam que existe uma tendência à elevação do número de casos. Torna-se necessário ainda, gerar conhecimentos atualizados sobre a distribuição geográfica das espécies dos caramujos hospedeiros intermediários do parasita para o planejamento e desenvolvimento das ações de controle e profilaxia ${ }^{6}$.

Assim sendo, o objetivo deste trabalho foi definir a prevalência da esquistossomose no povoado de Bom Gosto em Tutóia, Maranhão e detectar a ocorrência do hospedeiro intermediário.

\section{MÉTODOS}

Local

O presente estudo foi realizado de maio a outubro de 2008, em um povoado chamado Bom Gosto. Esta localidade fica localizada a $9 \mathrm{~km}$ de distância do município de Tutóia no Estado do Maranhão e possui 1.826 habitantes distribuídos em 495 residências.

\section{Procedimentos}

Foi realizado um levantamento dos dados referentes a amostras de fezes coletadas pela 
Secretaria de Saúde e Fundação Nacional de Saúde (FUNASA) que abrangeram 1.421 habitantes. A análise das mesmas foi realizada pelo método de Kato-Katz preconizado pela Organização Mundial de Saúde (OMS).

Posteriormente, caramujos foram coletados no Rio Bom Gosto, com o objetivo de determinar a ocorrência ou não do hospedeiro intermediário do parasita e verificar se estavam infectados pelo mesmo. As coletas ocorreram sempre no período da manhã, das 6 às 9 horas, no final de cada mês, sendo 10 em cada visita, totalizando 60 caramujos. Os espécimes eram colocados em vasilhame de vidro com água e transportados até o laboratório para diagnóstico de contaminação parasitária e identificação da espécie. O método utilizado foi o de esmagamento e em seguida foram observados ao microscópio.

\section{Considerações éticas}

Atendendo à resolução no 196/96 do Conselho Nacional de Saúde, que trata das normas para pesquisa envolvendo seres humanos, este projeto teve seu protocolo aprovado pelo Comitê de Ética em Pesquisa da Universidade Federal do Piauí.

\section{RESULTADOS}

Em 2008, foram realizados exames parasitológicos em aproximadamente $78 \%$ da população (1.421 habitantes). Destes, apenas 45 amostras foram positivas, tendo-se o percentual de 3,2\% de prevalência de esquistossomose na população estudada. Os dados epidemiológicos individuais não foram disponibilizados pela FUNASA.

Todos os caramujos foram identificados como sendo da espécie Biomphalaria glabrata. Dos espécimes examinados, 8,3\% encontravam-se infectados por S. mansoni, em julho, agosto e outubro (Tabela 1).

TABELA 1 - Valores absolutos de caramujos da espécie Biomphalaria glabrata infectados com Schistosoma mansoni coletados do Rio Bom Gosto de maio a outubro de 2008 .

\begin{tabular}{lcc}
\hline \multirow{2}{*}{ Mês } & \multicolumn{2}{c}{ Caramujos $\left(\mathrm{n}^{\circ}\right)$} \\
\cline { 2 - 3 } & coletados & positivos \\
\hline Maio & 10 & 0 \\
Junho & 10 & 0 \\
Julho & 10 & 2 \\
Agosto & 10 & 1 \\
Setembro & 10 & 0 \\
Outubro & 10 & 2 \\
\hline Total & $\mathbf{6 0}$ & $\mathbf{5}$ \\
\hline
\end{tabular}

\section{DISCUSSÃO}

A prevalência determinada pela Secretaria de Saúde do município de Tutóia indica uma baixa ocorrência de esquistossomose no povoado. Estes índices foram inferiores aos encontrados na Baixada Ocidental Maranhense ${ }^{7}$ e em outros estados do Nordeste como Pernambuco $^{3}$ e Bahia ${ }^{4}$. Mas foram superiores aos relatados em Bambuí, região Centro Oeste de Minas Gerais 5 .

Segundo estudos realizados anteriormente, a maior prevalência ocorre em crianças e adolescentes tanto em áreas de baixa prevalência como São Paulo ${ }^{8}$, quanto em áreas de média ${ }^{9}$ e alta prevalência ${ }^{3}$ relatadas em diferentes áreas do estado de Pernambuco. Dias e $\operatorname{cols}^{8}$, sugere que estas faixas etárias sejam as mais acometidas devido à presença do lazer como principal fator de risco. Aliada a este fato, sabe-se que os adultos desenvolvem imunidade contra este parasito, e os indivíduos que compõem esta parcela da população tem uma menor exposição aos fatores de risco ${ }^{10}$, diminuindo assim a prevalência da esquistossomose nesta faixa etária.

Foi relatado que os resquícios de material fecal na região anal de pessoas infectadas ainda podem ser capazes de contaminar a água durante a recreação ou a natação, pois os ovos podem sobreviver por um longo tempo em um ambiente úmido, como a região perianal ${ }^{11}$. Na região de estudo, é hábito da população divertir-se, bem como lavar roupas e banhar-se nos rios que margeiam as comunidades locais. Assim sendo, estes fatos facilitam a transmissão da doença no povoado de Bom Gosto.

O método de Kato-Katz é o preconizado pela Organização Mundial de Saúde para a detecção do parasita S. mansoni ${ }^{12}$. No entanto, sabe-se que o mesmo não é o melhor para determinar a prevalência da esquistossomose. Sua eficácia depende do nível de ocorrência da doença e da intensidade da infecção nos indivíduos parasitados ${ }^{13}$. Quando a segunda condição é baixa, a sensibilidade diagnóstica da técnica é diminuída, assim como em áreas pouco endêmicas ${ }^{14}$. A incorporação de testes sorológicos para a vigilância epidemiológica em zonas de transmissão pequena tenta compensar a subestimação da prevalência baseado apenas no diagnóstico parasitológico ${ }^{15}$.

No rio que banha a região, foram encontrados moluscos infectados pelo parasita S. mansoni, pertencentes à espécie B. glabrata, um dos seus hospedeiros intermediários. Esta espécie também foi encontrada em coleções hídricas do Estado de São Paulo. O conhecimento da presença de espécies dos caramujos transmissores de S. mansoni serve de suporte às investigações que visam o esclarecimento da origem dos casos humanos diagnosticados e notificados, a partir de inquéritos coprológicos ou dos exames de rotina da rede básica de saúde. Esta atividade é muito importante, pois a notificação sucessiva de casos humanos supostamente autóctones em uma dada localidade ou município, facilita a descoberta e o combate aos focos do parasita ${ }^{6}$. Esses aspectos malacológicos, associados aos padrões de contato com a água exibidos pelos moradores, tornam infrutíferas as tentativas de controle da doença baseadas apenas na quimioterapia ${ }^{3}$.

O povoado Bom Gosto é uma região de baixa prevalência da esquistossomose mansônica. A área investigada tem todos os componentes da cadeia epidemiológica de S. mansoni, explicando a ocorrência da doença na região. Sugere-se a implementação de políticas públicas de saúde a fim de impedir a sua disseminação. A utilização de melhores técnicas diagnósticas poderia estimar melhor a real prevalência da esquistossomose no estado do Maranhão.

\section{CONFLITO DE INTERESSE}

Os autores declaram não haver nenhum tipo de conflito de interesse no desenvolvimento do estudo.

\section{REFERÊNCIAS}

1. Katz N, Peixoto SV. Análise crítica da estimativa do número de portadores de esquistossomose mansoni no Brasil. Rev Soc Bras Med Trop 2000; 33:303-308.

2. Ferreira LA, Lima FLC, Anjos MRO, Costa JML. Forma tumoral encefálica esquistossomótica: apresentação de um caso tratado cirurgicamente. Rev Soc Bras Med Trop 1998; 31:89-93. 
3. Moza PG, Pieri OS, Barbosa CS, Rey L. Fatores sócio-demográficos e comportamentais relacionados à esquistossomose em uma agrovila da zona canavieira de Pernambuco, Brasil. Cad Saude Publica 1998; 14:107-115.

4. Bina JC, Prata A. Esquistossomose na área hiperendêmica de Taquarendi. I - Infecção pelo Schistosoma mansoni e formas graves. Rev Soc Bras Med Trop 2003; 36:211-216

5. Rocha RS, Silva JG, Peixoto SV, Caldeira RL, Firmo JOA, Carvalho OS. Avaliação da esquistossomose e de outras parasitoses intestinais, em escolares do município de Bambuí, Minas Gerais, Brasil. Rev Soc Bras Med Trop 2000; 33:431-436.

6. Teles HMS. Distribuição geográfica das espécies dos caramujos transmissores de Schistosoma mansoni no Estado de São Paulo. Rev Soc Bras Med Trop 2005; 38:426-432.

7. Cutrim RNM, Chieffi PP, Moraes JC. Schistosomiasis mansoni in the "Baixada Ocidental Maranhense”, state of Maranhão, Brazil: cross-sectional studies performed in 1987 and 1993. Rev Inst Med Trop Sao Paulo 1998; 40:165-172.

8. Dias LCS, Glasser CM, Marçal-Jr O, Bonesso PIP. Epidemiologia da Esquistossomose Mansônica em Área de Baixa Endemicidade. Cad Saude Publica 1994; 10:254-260

9. Barbosa CS, Barbosa FS. Padrão epidemiológico da esquistossomose em comunidade de pequenos produtores rurais de Pernambuco, Brasil. Cad Saude Publica 1998; 14:129-137.

10. Kabatereine NB, Vennervald BJ, Ouma JH, Kemijumbi J, Butterworth AE, Dunne DW. Adult resistance to schistosomiasis mansoni: age-dependence of reinfection remains constant in communities with diverse exposure patterns. Parasitology 1999; 118:101-105.

11. Sow S, Polman K, Vereecken K, Vercruysse J, Gryseels B, Vlas SJ. The role of hygienic bathing after defecation in the transmission of Schistosoma mansoni. Trans Rev Soc Trop Med Hyg 2008; 102:542-547.

12. World Health Organization. Bench AIDS for the diagnosis of intestinal parasites. World Health Organization, Geneva; 1994.

13. Berhe N, Medhina G, Erkoa B, Smith T, Gedamu S, Bereded D, et al. Variations in helminth faecal egg counts in Kato-Katz thick smears and their implications in assessing infection status with Schistosoma mansoni. Acta Trop 2004; 92: 205-212.

14. Enk MJ, Lima ACL, Drummond SC, Schall VT, Coelho PMZ. The effect of the number of stool samples on the observed prevalence and the infection intensity with Schistosoma mansoni among a population in an area of low transmission. Acta Trop 2008; 108:222-228.

15. Noya BA, Ruiz R, Losada S, Colmenares C, Contreras R, Cesari IM, et al. Detection of schistosomiasis cases in low-transmission areas based on coprologic and serologic criteria: The Venezuelan experience. Acta Trop 2007; 103:41-49. 